\title{
Review
}

\section{A Fresh Look at Huntingtin mRNA Processing in Huntington's Disease}

\author{
Lindsay Romo ${ }^{\mathrm{a}, *}$, Emily S. Mohn ${ }^{\mathrm{b}}$ and Neil Aronin ${ }^{\mathrm{a}, \mathrm{b}, *}$ \\ ${ }^{a}$ Department of Medicine, University of Massachusetts Medical School, Worcester, MA, USA \\ ${ }^{\mathrm{b}}$ RNA Therapeutics Institute, University of Massachusetts Medical School, Worcester, MA, USA
}

\begin{abstract}
Huntington's disease (HD) is an inherited neurodegenerative disorder caused by a mutation that expands the polyglutamine (CAG) repeat in exon 1 of the huntingtin (HTT) gene. Wild-type HTT protein interacts with other proteins to protect cells against toxic stimuli, mediate vesicle transport and endocytosis, and modulate synaptic activity. Mutant HTT protein disrupts autophagy, vesicle transport, neurotransmitter signaling, and mitochondrial function. Although many of the activities of wild-type HTT protein and the toxicities of mutant HTT protein are characterized, less is known about the activities of $H T T$ mRNA. Most putative HD therapies aim to target mutant HTT mRNA before it is translated into the protein. Therefore, it is imperative to learn as much as we can about how cells handle both wild-type and mutant HTT mRNA so that effective therapies can be designed. Here, we review the structure of wild-type and mutant $H T T$ mRNA, with emphasis on their alternatively polyadenylated or spliced isoforms. We then consider the abundance of $H T T$ mRNA isoforms in HD and discuss the potential implications of these findings. Evidence in the review should be used to guide future research aimed at developing mRNA-lowering therapies for HD.
\end{abstract}

Keywords: Huntington disease, mRNA, 3’UTR, alternative splicing, RNA 3' End Processing

\section{HTT MRNA IS PROCESSED INTO SEVERAL ALTERNATIVELY POLYADENYLATED ISOFORMS THAT CHANGE THEIR ABUNDANCE IN HD}

Experimental Huntington's disease (HD) therapies target mutant HTT mRNA before it is processed into the toxic HTT protein [1-4]. Therefore characterization of HTT mRNA alternatively polyadenylated isoforms may inform therapeutic design. Cleavage and polyadenylation of nascent mRNAs at the end

\footnotetext{
*Correspondence to: Lindsay Romo, University of Massachusetts Medical School, Department of Medicine, 55 Lake Ave. North, Worcester, MA 01655, USA. E-mail: lindsay.romo@umassmed.edu and Neil Aronin, University of Massachusetts Medical School, Department of Medicine, 55 Lake Ave. North, Worcester, MA 01655, USA. Tel.: +1 508856 3239; E-mail: neil.aronin@umassmed.edu.
}

of transcription is a critical step in mRNA maturation and is directed by nucleic acid motifs in the $3^{\prime}$ untranslated region (UTR) of the transcript [5]. One of the motifs is a hexanucleotide sequence called a polyA signal. The canonical polyA signal is AAUAAA, but other nucleotide variations can elicit cleavage and polyadenylation at lower efficiency [6-8]. Most mammalian mRNAs possess multiple cleavage and polyadenylation sites that give rise to $\mathrm{mRNA}$ isoforms of varying $3^{\prime}$ UTR lengths, a phenomenon termed alternative polyadenylation [9]. The choice of cleavage site depends on the transcription rate and the concentration of RNA binding proteins in the nucleus [10]. The length of the resulting $3^{\prime}$ UTR ultimately impacts the metabolism of each respective mRNA isoform (for in-depth review, see reference 11) [11]. Briefly, regulatory factors bind cis elements in the $3^{\prime}$ UTR to determine the localization and stability of 
the mature transcript $[5,11-13]$. For example, brainderived neurotrophic factor $(B D N F)$ is transcribed into two predominant mRNA 3'UTR isoforms. The longer isoform is localized to dendrites, where it plays a role in long-term potentiation [14]. The shorter isoform stabilizes mRNA in cell bodies after depolarization $[14,15]$. The stability of the mature transcript is dictated by the length of the $3^{\prime}$ UTR via interactions with microRNA (miRNA) and RNA binding proteins $[16,17]$. miRNAs target and destabilize mRNAs, and most miRNA sites are located in the $3^{\prime} \mathrm{UTR}$ after the first polyA site [16]. In T cells, mRNAs with extended 3'UTRs have twice as many miRNA target sites and produce less protein than mRNAs with shorter 3'UTRs [18]. Modulation of mRNA stability by RNA binding proteins also leads to changes in the amount of protein translated [19]. In yeast, deletions in many RNA binding proteins result in decreased mRNA steady-state levels [20]. Thus, alternative polyadenylation can affect mRNA activity, stability, and translation [21].

Three HTT mRNA 3'UTR isoforms are produced via alternative cleavage and polyadenylation at three different sites (Fig. 1A) [22, 23]. The longest mRNA isoform is 13.7 kilobases, the mid-length isoform is 12.5 kilobases, and the shortest isoform is 10.3 kilobases [22, 23]. All three isoforms are conserved in mice; conservation in other animals is unknown [23]. The long HTT mRNA isoform polyA signal, ATTAAA, has higher in vitro cleavage and polyadenylation rates than the short (AGTAAA) and mid-length (AAUGAA) isoform signals [6]. However, the relative abundance of these mRNA isoforms differs across tissues [22, 23]. For example, the long isoform is more abundant in the brain while the short isoform predominates in peripheral tissues such as liver and muscle [22, 23].

The abundance of HTT mRNA $3^{\prime} \mathrm{UTR}$ isoforms changes in HD. Although all three isoforms are found in HD and control brains, the steady-state abundance of these mRNA isoforms differs in fibroblasts and brain tissue of HD patients compared to controls [23]. Isoform changes appear to be tissue-specific and arise from both HTT alleles [23]. An example of this isoform change in the human motor cortex is illustrated in Fig. 1B. The abundance of the HTT short and mid-length mRNA isoforms in HD motor cortex is approximately double that in control, although the isoforms remain of lower abundance (12\% of total HTT mRNA) than the long isoform. It is possible that this observed shift in abundance is due to increased neuronal death in HD, which alters the cell population in the brain. However, mRNA expression has been shown to be comparable in HD caudate tissue and HD caudate neurons, despite extensive neuronal loss [24]. Moreover, the aforementioned isoform study used motor cortex from grade 1 and 2 HD brains, in which neuronal loss is limited [23, 25-27].

The mechanism underlying mRNA 3'UTR isoform alterations in HD is not established. However, one study determined that knockdown of an RNA binding protein, CCR4-NOT transcription complex subunit 6 (CNOT6), in human control fibroblasts resulted in HTT mRNA $3^{\prime}$ UTR isoform amounts similar to those in HD fibroblasts [23]. Furthermore, CNOT6 expression is decreased in HD patient motor cortex [23]. Taken together, these findings suggest that altered expression of certain RNA binding proteins in HD may contribute to mRNA isoform abundance changes. In addition to CNOT6, three $3^{\prime}$ end processing factors are differentially expressed in human HD motor cortex: CPSF2, PCBP2, and THOC5 [23]. These proteins should also be explored as potential causes of HTT $3^{\prime} \mathrm{UTR}$ isoform shifts in HD.

\section{IMPLICATIONS FOR ALTERED ABUNDANCE OF HTT MRNA 3'UTR ISOFORMS IN HD}

HTT mRNA $3^{\prime} \mathrm{UTR}$ isoforms are translated into identical proteins. However, the different $H T T 3^{\prime}$ UTR isoforms have distinct localizations, stabilities, polyA tail lengths, and different sites for RNA binding proteins and microRNAs [23]. In wild-type and HD fibroblasts, the mid-length mRNA isoform is more abundant in the cytoplasm whereas the short and long isoforms are more abundant in the nucleus [23]. The short isoform has the longest half-life, followed by the mid-length, then long isoform [23]. This may be because the polyA tail length of the short isoform is 50 nucleotides longer than that of the mid-length and long isoforms, both of which have more microRNA binding sites than the short isoform [23]. Further work should investigate whether rates of translation significantly differ among the mRNA isoforms. Taken together, these findings suggest HTT $3^{\prime}$ UTR shifts in HD impact HTT mRNA and protein load.

The impact of altering mRNA and protein load due to $3^{\prime}$ UTR isoform shifts has been documented in several other diseases. In patients with non-anterior uveitis, a single nucleotide polymorphism in the interferon regulatory factor 5 (IRF5) mRNA creates a polyadenylation site, resulting in a short mRNA that is associated with the development of macular 


\section{A An overview of HTT mRNA 3' end processing}

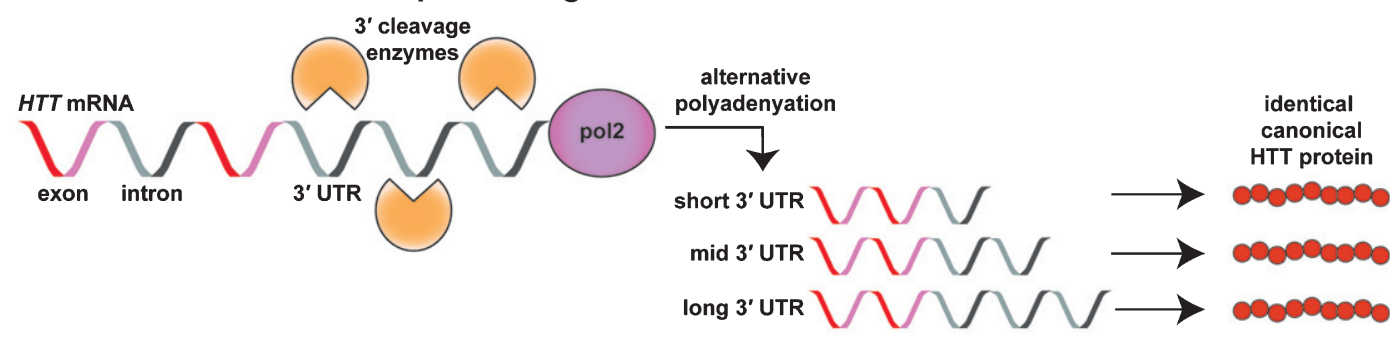

\section{B HTT mRNA 3' end processing in normal versus HD motor cortex}

\section{Normal}

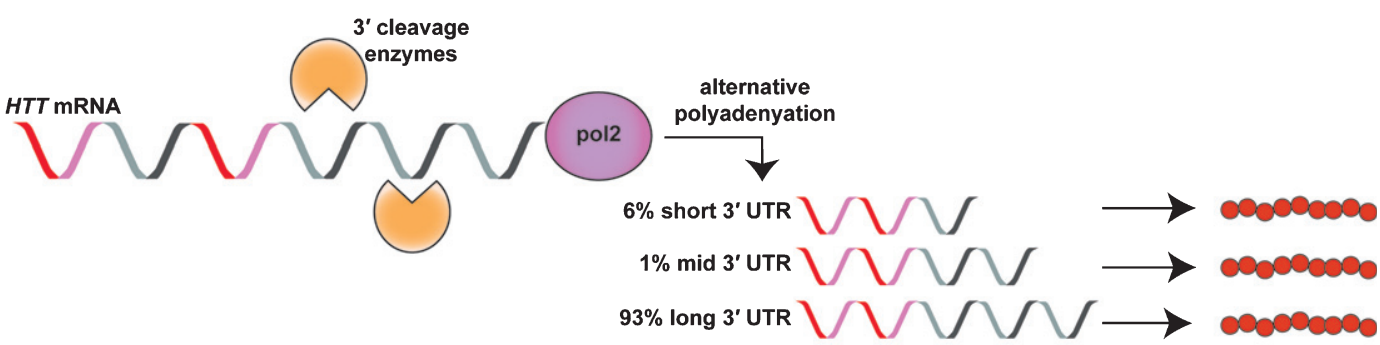

HD

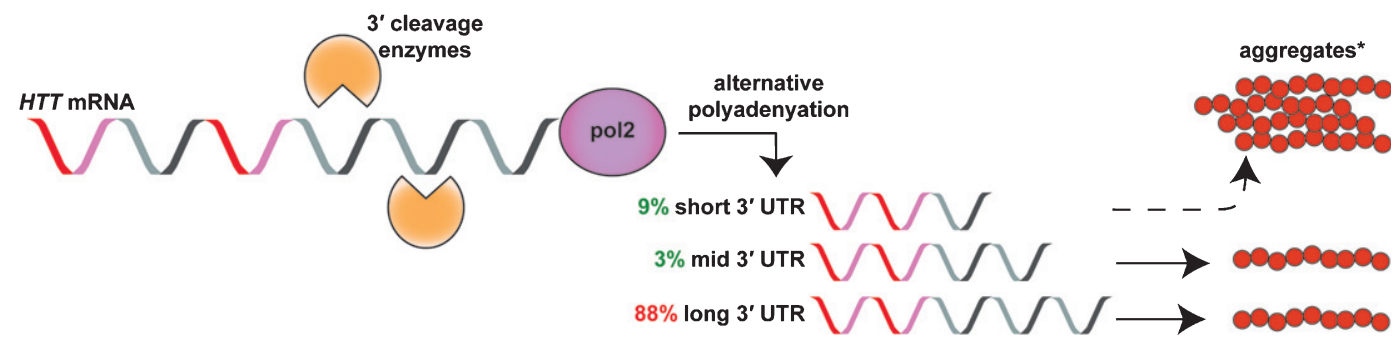

Fig. 1. HTT mRNA is processed into several alternatively polyadenylated isoforms that change their abundance in HD versus normal human motor cortex. A) The HTT gene is transcribed into mRNA by RNA polymerase 2 (pol2). During transcription, nascent HTT mRNA can be alternatively cleaved and polyadenylated at three putative polyA sites in its $3^{\prime} \mathrm{UTR}$ producing a $10.3 \mathrm{~kb}$ (short), $12.5 \mathrm{~kb}$ (mid), or $13.7 \mathrm{~kb}$ (long) transcript $[22,23]$. These alternatively polyadenylated isoforms are translated into the canonical HTT protein. B) In HD patient motor cortex, the amount of the short and mid $3^{\prime} \mathrm{UTR}$ isoforms increases relative to the long isoform [23]. *The short $3^{\prime} \mathrm{UTR}$ isoform forms more aggregates than the long isoform in vitro [30], suggesting altered isoform abundance may have an impact on the formation of abnormal protein-protein interactions.

edema [28]. Similarly, single nucleotide polymorphisms associated with Parkinson's disease promote formation of the longest mRNA isoform of alpha synuclein [29]. This results in accumulation of the protein and localization to mitochondria, both in vitro and in Parkinson's patient brains, potentially causing pathology [29]. In HD, the increase in the HTT short mRNA isoform in patient motor cortex may contribute to toxicity. In vitro experiments have shown that cells transfected with the more-stable short HTT mRNA isoform have more aggregates than those transfected with the long HTT isoform, suggesting that $H T T 3^{\prime}$ UTR isoforms differentially affect the formation of abnormal protein-protein interactions [30]. Further studies should investigate whether this affects
HD pathogenesis. Finding one mRNA isoform to be more pathogenic than the others may provide new therapeutic strategies for HTT RNA silencing. However, the exact role of $H T T 3^{\prime} \mathrm{UTR}$ isoform changes in HD remains to be established.

\section{HTT MRNA IS PROCESSED INTO SEVERAL SPLICE ISOFORMS THAT MAY CHANGE THEIR ABUNDANCE IN HD}

mRNA splicing occurs concurrently with transcription and polyadenylation. During this process, splicing proteins interact with $\mathrm{cis}$-factors to exclude introns and join exons. Like polyadenylation, the 
splicing process is influenced by several factors, including RNA binding protein concentration, RNA-RNA base-pairing, and the chromatin environment, to produce different transcripts from the same gene [31]. In fact, alternative splicing and polyadenylation can be co-regulated by certain RNA binding proteins [32]. Heterogeneous ribonucleoprotein $\mathrm{H}$ (HNRNPH1), a mediator of mRNA alternative splicing, also suppresses cryptic polyA sites, and two $3^{\prime}$ end formation proteins-cleavage and polyadenylation specificity factor (CPSF) and Symplekin (SYMPK) - also regulate splicing of internal exons [33, 34]. However, unlike alternative polyadenylation, alternative splicing results in mRNA isoforms that are translated into different protein products, which may have unique functions or post-translational modifications [31]. For example, upon neuronal activation some $B D N F$ splice variants are localized to dendrites in rat hippocampus whereas others are restricted to the soma, allowing for targeted expression of the BDNF protein [35].

HTT nascent mRNA undergoes alternative splicing. The resulting splice isoforms are of low abundance compared to the most common (canonical) splice isoform, and the translation and function of most HTT splice variants has not been established (Fig. 2A) [36]. In wild-type mouse brains, HTT can be alternatively spliced to lack either exon 28 , exon 29 or exons 34-44, or spliced to retain a portion of intron 28; the mRNA isoforms lacking exon 28/29 are also present in human brains [37, 38]. Isoforms lacking exon $28 / 29$ or retaining intron 28 are most abundant in cerebellum and least abundant in the striatum [37]. The mRNA isoform lacking exon 29 is expressed at lower levels in HD Q150 knock-in mice compared to wild-type mice (Fig. 2B, 20\% lower) [37].

Another study identified five more HTT mRNA splice isoforms in human embryonic stem cell lines differentiated into neural precursors [39]. The expression of the mRNA isoforms ranged from $0.8 \%$ to $13.6 \%$ of the most common transcript. Only one of these mRNA splice isoforms has also been detected in human tissues. This isoform is hominid-specific and contains a previously undiscovered exon between exons 41 and 42, dubbed 41b [39]. Eighteen additional alternatively spliced isoforms with abundances ranging from $1 \%$ to $7 \%$ of the canonical transcript have been identified using high-throughput sequencing of control and HD human brain tissue [36]. However, this study did not attempt to compare HD to control brains, so it is unclear if the abundance of these HTT splice variants changes in HD patients. Finally, PCR and sequencing of $H T T$ cDNA from human brain identified twenty-two mRNA splice variants, including $41 \mathrm{~b}$ and a transcript lacking exons 4-6 [40]. However, the study found no difference between the abundance of these mRNA splice isoforms in HD and control human brains [40].

\section{IMPLICATIONS FOR HTT MRNA SPLICE ISOFORMS IN HD}

Many genes alter their splicing in HD patient brains, potentially due to sequestration of the splicing factor MBNL1 by mutant HTT mRNA, or to altered expression of other RNA binding proteins in HD [41, 42]. The abundance of HTT mRNA splice isoforms may also change in HD. If the isoform lacking exon 29 is found to be of lower abundance in HD patients as it is in HD mice, it may have functional consequences. Exon 29 contains a putative binding site for the Tap protein, which is involved in nuclear export of RNAs [37]. Loss of this binding site could result in nuclear retention of the HTT transcripts. However, this mRNA isoform is of such low abundance in human brain that it could only be detected after several rounds of amplification and could not be quantified via PCR [37]. Another HTT splice variant of interest is the isoform lacking exons 4-6 identified in human brain. Exons 4-6 contain a nuclear localization signal, two post-translational modification sites, and a predicted binding site for a nuclear localization protein [40]. Thus, a HTT protein lacking these domains may be localized differently than canonical HTT protein, which could affect pathology [43]. Evidence for a role of alternative splicing in disease pathology has been observed in tauopathies. Alternative splicing of the microtubule associated protein tau gene results in six isoforms; shifting the ratio of two of these isoforms leads to neurodegeneration in frontotemporal dementia [44]. For HD, it has not been established whether HTT mRNA splice isoforms are translated or whether they impact HD pathology. Future studies should investigate whether HTT mRNA splice variants, particularly the isoforms lacking either exon 28 or exons 4-6, play a role in HD.

\section{AN ABERRANT HTT SPLICE ISOFORM FOUND IN HD MAY BE PATHOGENIC}

Aberrant splicing refers to an abnormal variation in the splicing process and has been shown to contribute 
A An overview of HTT mRNA alternative splicing

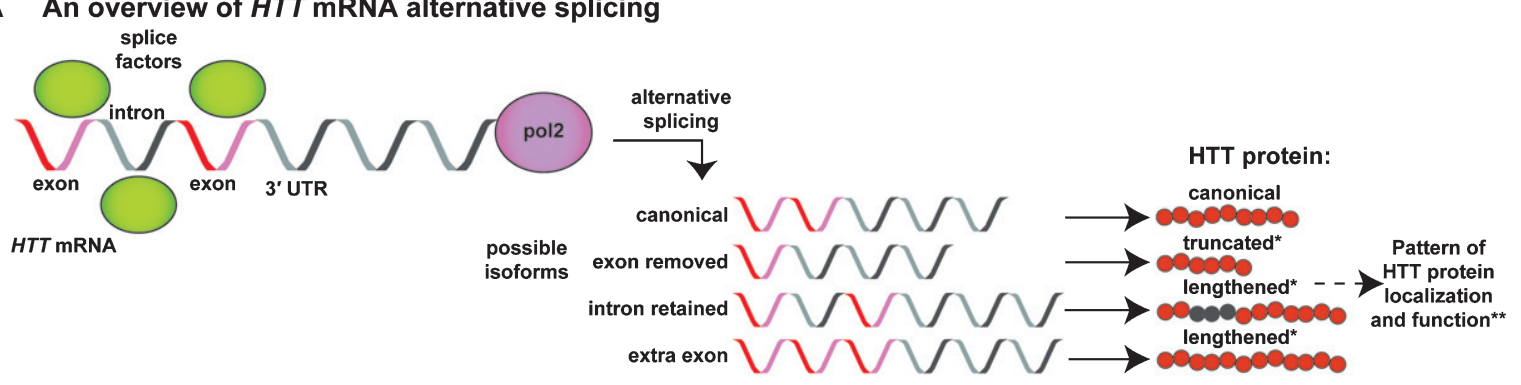

B Some splice variants change abundance in cerebellum of Q150 HD knock-in mouse model WT mouse

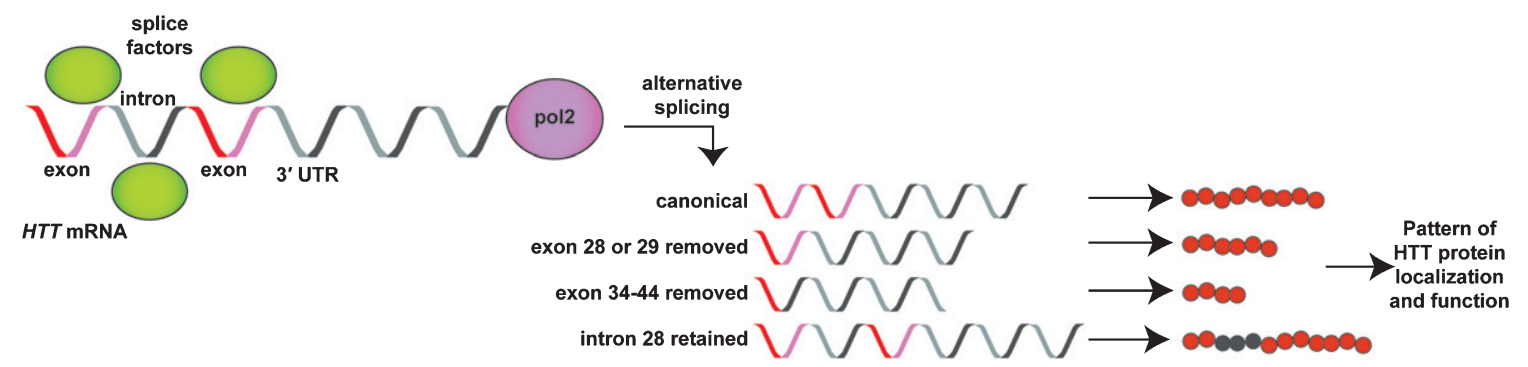

Q150 HD mouse

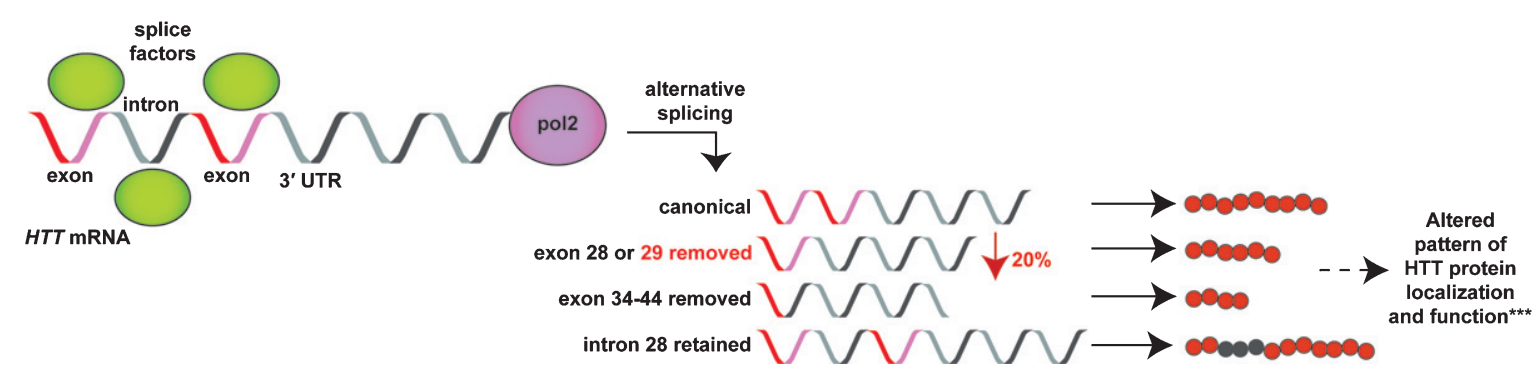

Fig. 2. HTT is transcribed into several splice isoforms in normal and HD tissues. A) In normal and HD cells, HTT mRNA can be alternatively spliced to lack an exon(s), producing a shorter mRNA and protein than the canonical transcript, or to include an extra exon or retain an intron, producing longer mRNA and protein [36-40]. *The translation of these isoforms has yet to be demonstrated. ${ }^{* *}$ However, if translated, splice variant protein products may have different localization and function. For example, a splice isoform of similar abundance in patient and control brains that excludes exons 4-6 lacks a nuclear localization signal [40]. B) In wild-type mice, HTT can be alternatively spliced to lack exon 28, 29, 24-44, or to retain intron 28 [37, 38]. These isoforms are of the same or unknown abundance in HD mouse models except the variant lacking exon 29, which is of decreased abundance in Q150 HD knock-in mice [37]. *** Decreased abundance may have an impact on HTT localization and function, as exon 29 has a binding site for a protein involved in RNA nuclear export (Tap) [37].

to disease [31]. In spinal motor atrophy, both copies of the survival motor neuron 1 (SMN1) gene are deleted, leaving the duplicate $S M N 2$ gene as the only source of the SMN protein $[45,46]$. A frequent nucleotide variant in the $S M N 2$ gene at a splicing enhancement site leads to missplicing and an unstable truncated protein that exacerbates the disease [47]. Aberrant splicing may also occur in HD. An elegant study published by the Bates group characterized one truncated HTT mRNA splice isoform that may be pathogenic [48]. In HD patients and mouse models, but not controls, $H T T$ is misspliced and polyadenylated at a cryptic polyadenylation signal in intron 1 , producing a truncated transcript [48] (Fig. 3). In mice, production of the aberrant transcript is correlated with the CAG repeat number. Although quantitation of this mRNA isoform is difficult because of its high GC-content and stable secondary structure, the truncated transcript has been detected via $3^{\prime}$ RACE (rapid amplification of cDNA ends) in HD patient, but not control, fibroblasts and brain tissue [48-50]. This study also observed higher levels of $H T T$ mRNA containing intron 1 in patient motor cortex and hippocampus compared to controls [50]. 


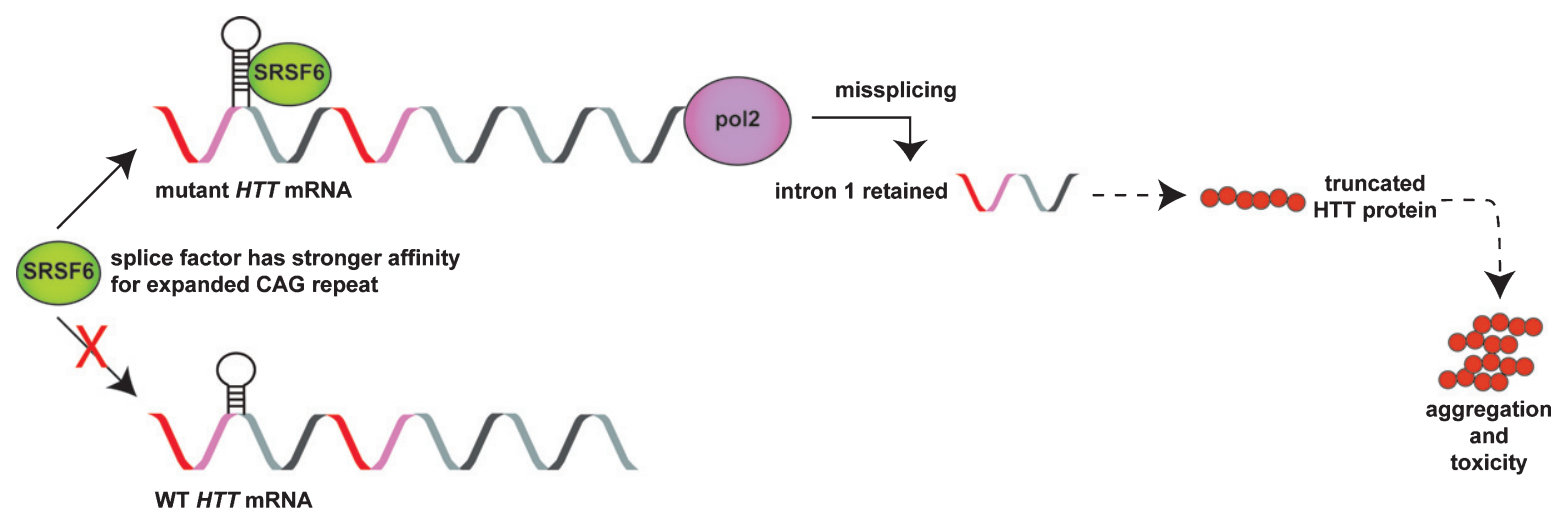

Fig. 3. HTT is alternatively spliced into a truncated isoform in HD cells. In HD fibroblasts and brain tissue, the splicing factor SRSF6 processes mutant $H T T$ mRNA into a third alternatively polyadenylated splice isoform that terminates in intron 1 . This isoform may be translated into the pathogenic N-terminal HTT protein prone to aggregation and toxicity [48-50].

Production of the transcript may be controlled by the serine/arginine-rich splicing factor 6 (SRSF6), which binds to expanded mutant $H T T$ mRNA with a higher affinity than to wild-type mRNA (Fig. 3). A stop codon at the very beginning of intron 1 precludes the production of an intron- 1 specific antibody, but immunoprecipitation of $\mathrm{N}$-terminal HTT detected a protein of the appropriate size in HD mouse models that was not detected in controls [48]. The protein could not be immunoprecipitated from HD patient brains, possibly because it is highly prone to aggregation [50]. It has not been established if this protein represents translation of the truncated splice isoform or post-translational cleavage of the full-length protein. The association of this splice isoform with disease and the toxicity of N-terminal fragments of the HTT protein suggest that it could contribute to HD pathology. If the potential link between this splice isoform and disease can be established, intron 1 may be an exciting new therapeutic target.

\section{CONCLUSIONS}

For decades, HD research has focused on the toxic mutant HTT protein. However, recent studies suggest HTT mRNA may also influence cellular toxicity. There are multiple alternatively spliced or polyadenylated isoforms of mutant HTT mRNA. The studies detailed in this review suggest some isoforms of HTT mRNA may be more toxic than others, either independently or because of their protein products. However, research on HTT mRNA structure, activity, and toxicity is in its infancy with most of the studies described in this review being correlative. Differential handling and processing of HTT mRNA may be a contributor to pathology, or it may be a result of pathology. Regardless, mutant HTT mRNA is an ideal target for HD therapeutics because it is translated into the pathogenic protein, and thus should continue to be rigorously investigated [4]. This will aid in the development of more efficient and effective HD therapies designed to target specific mutant HTT mRNA isoforms. For instance, if the truncated HTT mRNA isoform is found to be pathogenic, it could be targeted with interfering RNAs complementary to intron 1 . If the short $3^{\prime}$ UTR HTT isoform is found to be toxic, the proximal polyA site could be disrupted in the HTT gene. Moreover, knowledge of the differential pathogenicity and localization of HTT mRNA isoforms may suggest cellular compartments that could be targeted by RNA-lowering therapies. Thus, it is critical that future research address the current gaps in knowledge regarding differential toxicity of $H T T$ mRNA isoforms in an effort to further characterize HD pathogenesis and guide recommendations for HTT mRNA-lowering therapies

\section{CONFLICT OF INTEREST}

The authors have no conflict of interest to report.

\section{REFERENCES}

[1] Cattaneo E, Zuccato C, Tartari M. Normal huntingtin function: An alternative approach to Huntington's disease. Nat Rev Neurosci. 2005;6:919-30.

[2] Zuccato C, Valenza M, Cattaneo E. Molecular mechanisms and potential therapeutical targets in Huntington's disease. Physiol Rev. 2010;90:905-81.

[3] Harjes P, Wanker EE. The hunt for huntingtin function: Interaction partners tell many different stories. Trends Biochem Sci. 2003;28:425-33. 
[4] Aronin N, Difiglia M. Huntingtin-lowering strategies in Huntington's disease: Antisense oligonucleotides, small RNAs, and gene editing. Mov Disord. 2014;29:1455-61.

[5] Gruber AR, Martin G, Keller W, Zavolan M. Means to an end: Mechanisms of alternative polyadenylation of messenger RNA precursors. Wiley Interdiscip Rev RNA. 2014;5:183-96.

[6] Sheets MD, Ogg SC, Wickens MP. Point mutations in AAUAAA and the poly(A) addition site: Effects on the accuracy and efficiency of cleavage and polyadenylation in vitro. Nucleic Acids Res. 1990;18:5799-805.

[7] Proudfoot N, Brownlee G. Sequence at the 3'end of globin mRNA shows homology with immunoglobulin light chain mRNA. Nature. 1974;252:359-62.

[8] Proudfoot NJ. Ending the message: Poly (A) signals then and now. Genes Dev. 2011;25:1770-82.

[9] Derti A, Garrett-Engele P, Macisaac KD, Stevens RC, Sri$\operatorname{ram~S,~Chen~R,~et~al.~A~quantitative~atlas~of~polyadenylation~}$ in five mammals. Genome Res. 2012;22:1173-83.

[10] Shi Y. Alternative polyadenylation: New insights from global analyses. 2012:2105-17.

[11] Wang ET, Taliaferro JM, Lee JA, Sudhakaran IP, Rossoll W, Gross C, et al. Dysregulation of mRNA localization and translation in genetic disease. J Neurosci. 2016;36: 11418-26.

[12] Kislauskis EH, Singer RH. Determinants of mRNA localization. Curr Opin Cell Biol. 1992;4:975-8.

[13] Andreassi C, Riccio A. To localize or not to localize: mRNA fate is in 3'UTR ends. Trends Cell Biol. 2009;19:465-74.

[14] An JJ, Gharami K, Liao GY, Woo NH, Lau AG, Vanevski F, et al. Distinct role of long 3' UTR BDNF mRNA in spine morphology and synaptic plasticity in hippocampal neurons. Cell. 2008;134:175-87.

[15] Fukuchi M, Tsuda M. Involvement of the 3'-untranslated region of the brain-derived neurotrophic factor gene in activity-dependent mRNA stabilization. J Neurochem. 2010;115:1222-33.

[16] Legendre M, Ritchie W, Lopez F, Gautheret D. Differential repression of alternative transcripts: A screen for miRNA targets. PLoS Comput Biol. 2006;2:e43.

[17] Gebauer F, Preiss T, Hentze MW. From cis-regulatory elements to complex RNPs and back. Cold Spring Harb Perspect Biol. 2012;4:a012245.

[18] Sandberg R, Neilson JR, Sarma A, Sharp PA, Burge CB. Proliferating cells express mRNAs with shortened 3' untranslated regions and fewer microRNA target sites. Science. 2008;320:1643-7.

[19] Szostak E, Gebauer F. Translational control by 3'-UTRbinding proteins. Brief Funct Genomics. 2013;12:58-65.

[20] Hasan A, Cotobal C, Duncan CD, Mata J. Systematic analysis of the role of RNA-binding proteins in the regulation of RNA stability. PLoS Genet. 10:e1004684.

[21] Mueller AA, Cheung TH, Rando TA. All's well that ends well: Alternative polyadenylation and its implications for stem cell biology. Curr Opin Cell Biol. 2013;25:222-32.

[22] Lin B, Rommens JM, Graham RK, Kalchman M, MacDonald H, Nasir J, et al. Differential 3' polyadenylation of the huntington disease gene results in two mRNA species with variable tissue expression. Hum Mol Genet. 1993;2:1541-5.

[23] Romo L, Ashar-Patel A, Pfister E, Aronin N. Alterations in mRNA 3'UTR isoform abundance accompany gene expression changes in human Huntington's disease brains. Cell Rep. 2017;20:3057-70.

[24] Hodges A, Strand AD, Aragaki AK, Kuhn A, Sengstag T, Hughes G, et al. Regional and cellular gene expression changes in human Huntington's disease brain. Hum Mol Genet. 2006;15:965-77.

[25] Thu DC, Oorschot DE, Tippett LJ, Nana AL, Hogg VM, Synek BJ, et al. Cell loss in the motor and cingulate cortex correlates with symptomatology in Huntington's disease. Brain. 2010;133:1094-110.

[26] Vonsattel JP, Myers RH, Stevens TJ, Ferrante RJ, Bird ED, Richardson Jr EP. Neuropathological classification of Huntinqton's disease. J Neuropathol Exp Neurol. 1985;44:559-77.

[27] Rüb U, Hoche F, Brunt ER, Heinsen H, Seidel K, Del Turco $\mathrm{D}$, et al. Degeneration of the cerebellum in huntingtons disease (HD): Possible relevance for the clinical picture and potential gateway to pathological mechanisms of the disease process. Brain Pathol. 2013;23:165-77.

[28] Márquez A, Cánit MC, Cordero-Coma M, Ortego-Centeno $\mathrm{N}$, Adán A, Fonollosa A, et al. Two functional variants of IRF5 influence the development of macular edema in patients with non-anterior uveitis. PLoS One. 2013;8: 6-11.

[29] Rhinn H, Qiang L, Yamashita T, Rhee D, Zolin A, Vanti $\mathrm{W}$, et al. Alternative $\alpha$-synuclein transcript usage as a convergent mechanism in Parkinson's disease pathology. Nat Commun. 2012;3:1084.

[30] Xu H, An JJ, Xu B. Distinct cellular toxicity of two mutant huntingtin mRNA variants due to translation regulation. PLoS One. 2017;1-19.

[31] Lee Y, Rio DC. Mechanisms and regulation of alternative pre-mRNA splicing. Annu Rev Biochem. 2015;84: 291-323.

[32] Misra A, Green MR. From polyadenylation to splicing: Dual role for mRNA 3' end formation factors. RNA Biol. 2016;13:259-64.

[33] Misra A, Ou J, Zhu LJ, Green MR. Global promotion of alternative internal exon usage by mRNA 3' end formation factors. Mol Cell. 2014;58:819-31.

[34] Nazim M, Masuda A, Rahman MA, Nasrin F, Takeda JI1, Ohe $\mathrm{K}$, et al. Competitive regulation of alternative splicing and alternative polyadenylation by hnRNP $\mathrm{H}$ and CstF64 determines acetylcholinesterase isoforms. Nucleic Acids Res. 2017;45:1455-68.

[35] Chiaruttini C, Sonego M, Baj G, Simonato M, Tongiorgi E. BDNF mRNA splice variants display activity-dependent targeting to distinct hippocampal laminae. Mol Cell Neurosci. 2008;37:11-9.

[36] Labadorf AT, Myers RH. Evidence of extensive alternative splicing in post mortem human brain HTT transcription by mRNA sequencing. PLoS One. 2015;10:e0141298.

[37] Hughes AC, Mort M, Elliston L, Thomas RM, Brooks SP, Dunnett SB, et al. Identification of novel alternative splicing events in the huntingtin gene and assessment of the functional consequences using structural protein homology modelling. J Mol Biol. 2014;426:1428-38.

[38] Lin B, Nasir J, MacDonald H, Hutchinson G, Graham RK, Rommens JM, et al. Sequence of the murine Huntington disease gene: Evidence for conservation, alternate splicing and polymorphism in a triplet (CCG) repeat [corrected]. Hum Mol Genet. 1994;3:85-92.

[39] Ruzo A, Ismailoglu I, Popowski M, Haremaki T, Croft GF, Deglincerti A, et al. Discovery of novel isoforms of Huntingtin reveals a new hominid-specific exon. PLoS One. 2015;10:e0127687.

[40] Mort M, Carlisle FA, Waite AJ, Elliston L, Allen ND, Jones $\mathrm{L}$, et al. Huntingtin exists as multiple splice forms in human brain. J Huntingtons Dis. 2015;4:161-71. 
[41] Mykowska A, Sobczak K, Wojciechowska M, Kozlowski P, Krzyzosiak WJ. CAG repeats mimic CUG repeats in the misregulation of alternative splicing. Nucleic Acids Res. 2011;39:8938-51.

[42] Lin L, Park JW, Ramachandran S, Zhang Y, Tseng YT, Shen $\mathrm{S}$, et al. Transcriptome sequencing reveals aberrant alternative splicing in Huntington's disease. Hum Mol Genet. 2016;25:1-13.

[43] Gu X, Cantle JP, Greiner ER, Lee CY, Barth AM, Gao F, et al. N17 modifies mutant huntingtin nuclear pathogenesis and severity of disease in HD BAC transgenic mice. Neuron. 2015;85:726-41.

[44] Kar A, Kuo D, He R, Kar A, Kuo D, He R, et al. Tau alternative splicing and frontotemporal dementia. Tau alternative splicing and frontotemporal dementia. Alzheimer Dis Assoc Disord. 2005;19(Suppl 1):S29-36.

[45] Lefebvre S, Bürglen L, Reboullet S, Clermont O, Burlet $\mathrm{P}$, Viollet $\mathrm{L}$, et al. Identification and characterization of a spinal muscular atrophy-determining gene. Cell. 1995;80: $155-65$.
[46] Rochette CF, Surh LC, Ray PN, McAndrew PE, Prior TW, Burghes $\mathrm{AH}$, et al. Molecular diagnosis of non-deletion SMA patients using quantitative PCR of SMN exon 7. Neurogenetics. 1997;1:141-7.

[47] Cho S, Dreyfuss G. A degron created by SMN2 exon 7 skipping is a principal contributor to spinal muscular atrophy severity service A degron created by SMN2 exon 7 skipping is a principal contributor to spinal muscular atrophy severity. Genes Dev. 2010;438-42.

[48] Sathasivam K, Neueder A, Gipson TA, Landles C, Benjamin AC, Bondulich MK, et al. Aberrant splicing of HTT generates the pathogenic exon 1 protein in Huntington disease. Proc Natl Acad Sci U S A. 2013;110:2366-70.

[49] Gipson TA, Neueder A, Wexler NS, Bates GP, Housman D. Aberrantly spliced HTT, a new player in Huntington's disease pathogenesis. RNA Biol. 2013;10:1647-52.

[50] Neueder A, Landles C, Ghosh R, Howland D, Myers RH, Faull RLM, et al. The pathogenic exon 1 HTT protein is produced by incomplete splicing in Huntington's disease patients. Sci Rep. 2017;7:1307. 